\title{
OESOPHAGEAL DISEASE
}

\section{Endocinch therapy for gastro-oesophageal reflux disease: a one year prospective follow up}

\author{
Z Mahmood, B P McMahon, Q Arfin, P J Byrne, J V Reynolds, E M Murphy, D G Weir
}

Gut 2003;52:34-39

See end of article for authors' affiliations

\section{Correspondence to:} Professor D G Weir Department of Clinical Medicine, Trinity Centre for Health Sciences, St James's Hospital, Dublin 8, Ireland; dweir@tcd.ie

Accepted for publication 25 June 2002

\begin{abstract}
Background: Gastro-oesophageal reflux disease (GORD) is, in the main, treated with either proton pump inhibitor (PPI) drugs or a Nissen fundoplication operation. Recently, BARD developed Endocinch, a device used to place sutures just below the oesophagogastric junction (OGJ) to treat GORD.

Aim: To evaluate the long term benefit of the Endocinch technique in patients seen up to 12 months post procedure.

Patients: Twenty six patients with symptoms of GORD were recruited and had the procedure performed. Four patients were lost to follow up.

Methods: Twenty two patients completed their one year follow up. Pre procedure and post procedure (up to 12 months) assessments included symptom scoring (DeMeester), upper intestinal endoscopy, oesophageal manometry and 24 hour oesophageal $\mathrm{pH}$, and completion of quality of life (QOL) questionnaires.

Results: Mean age was 39 years (range 22-62). Heartburn symptom score was reduced from a mean value of 19.22 at baseline to 7.5 at 12 months $(n=22)(p<0.0001)$. Regurgitation score reduced from a mean of 2.27 at baseline to 0.86 at 12 months $(n=22)(p<0.001)$. Mean (SEM) pH DeMeester acid score was reduced from 44.1 (4.3) to 33.32 (4.73) $(p=0.028)$ at three month post procedure. Percentage upright acid exposure and number of reflux episodes were also reduced significantly. Use of PPIs was reduced by $64 \%$ at 12 months post procedure. All QOL assessments showed significant improvement $(p=0.01)$. All transient post procedure complaints resolved within 72 hours.

Conclusion: The Endocinch procedure is an effective and safe outpatient procedure that offers GORD patients significant improvement in symptomatology, QOL, and reduced requirements for PPIs over at least a one year period.
\end{abstract}

Abbreviations: GORD, gastro-oesophageal reflux disease; PPI, proton pump inhibitor; OGJ, oesophagogastric junction; $Q O L$, quality of life; LNF, laparoscopic Nissen fundoplication; BMI, body mass index; LOS, lower oesophageal sphincter; TLOSR, transient lower oesophageal sphincter relaxation. 
Table 1 Symptom scoring used to assess the severity of reflux symptoms

\begin{tabular}{ll}
\hline Heartburn severity visual analogue scale & $0=$ no discomfort \\
& $10=$ incapacitating pain \\
& $0=$ none \\
1 & $=$ occasional $(<3 /$ week) brief episodes controlled by \\
& antacids \\
2 & $=$ frequent $(3-5 /$ week $)$ \\
3 & $=$ daily $(6-7 /$ week $)$ \\
& $=$ none \\
1 & $=$ mild, occasional episodes, mostly postprandial and not \\
& predictable \\
2 & $=$ moderate $(3-5 /$ week), frequent episodes, predictable by \\
posture & $3=$ severe $(6-7 /$ week $)$, episodes interfering with work and \\
social activities & \\
Regurgitation symptom score &
\end{tabular}

discontinuing PPIs for 10 days and antacids for 48 hours before the test.

(iii) They agreed to be followed up for at least six months post procedure, which was extended to 12 months in most patients. They also agreed to have repeat endoscopy and 24 hour oesophageal pH studies three months afterwards.

Exclusion criteria were age $<18$ years, dysphagia, body mass index $(\mathrm{BMI})>40$, previous gastric or thoracic surgery, hiatus hernia $>2 \mathrm{~cm}$ in length, oesophageal stricture or varices, Barrett's oesophagus, patients on immunosuppressant drugs, and patients with contraindications for general surgery, as defined by Filipi and colleagues. ${ }^{15}$

\section{Patient assessment}

All patients gave written and informed consent to an independent observer who was not involved with the treatment. The procedure was explained and they were offered the alternatives of lifelong PPIs or surgical Nissen fundoplication. Those who consented had the severity of their GORD symptoms assessed when off medication, according to the symptom scoring scale shown in table 1. A similar detailed history of all symptoms was obtained at one, three, six, and 12 months post procedure. The quantity of PPIs consumed was noted. The QOLRAD quality of life questionnaire ${ }^{16}$ was completed independently by patients before the procedure at

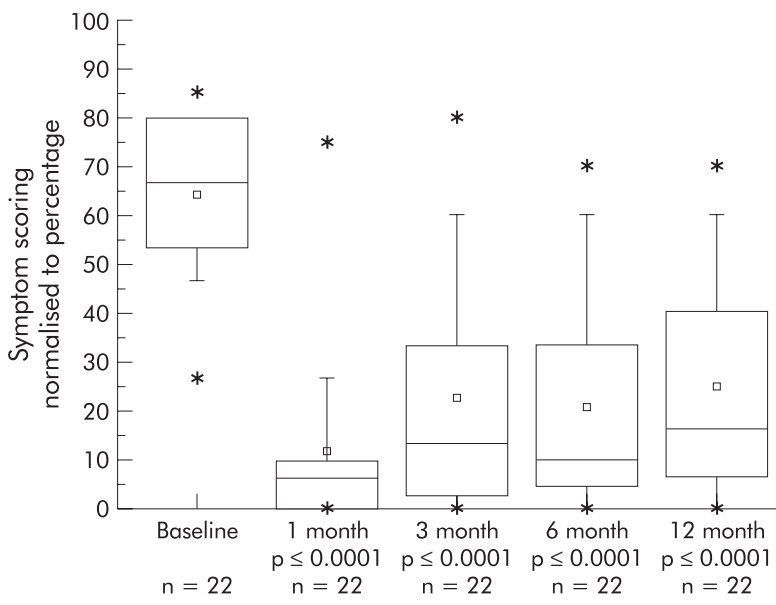

Figure 1 Symptom scoring of the patient data set, normalised to percentage, before (baseline) and one, three, six, and 12 months post procedure, where a maximum symptom score of 30 is represented as $100 \%$. The horizontal lines of the box denote the 25th, 50th, and 75th percentile values, where the 50th percentile is the median of the range of the data. The square symbol in the box denotes the mean. Error bars denote the 5th and 95th percentiles and asterisks the 0 and 100th percentiles. one, three, six, and 12 months post procedure. An independent observer who was not involved with the procedure over saw all symptom and QOLRAD assessments.

Upper gastrointestinal endoscopy was carried out before and three months post procedure, initially to establish the degree of oesophagitis present, the presence and size of any hiatus hernia, and to establish the position of the " $\mathrm{Z}$ " line. Endoscopy at three months assessed the integrity of the plications, their position, and the presence and degree of any oesophagitis.

\section{Oesophageal manometry}

Patients were investigated after an overnight fast. A four channel standard oesophageal manometric catheter was used (Mui Scientific, Ontario Canada) with lateral openings $5 \mathrm{~cm}$ apart and orientated radially around the circumference. A station pull through technique was employed. OGJ pressure was measured as end expiratory pressure at the point of respiratory reversal. Ten wet swallows were performed using $5 \mathrm{ml}$ boluses of water to record oesophageal body motility. Lower oesophageal sphincter (LOS) pressures and lengths were also recorded.

\section{pH monitoring}

Twenty hour $\mathrm{pH}$ monitoring was performed as described by DeMeester and colleagues. ${ }^{17}$ Patients discontinued PPIs for 10 days and antacids for 48 hours before $\mathrm{pH}$ monitoring. An antimony $\mathrm{pH}$ catheter (Medtronic, Copenhagen) was placed $5 \mathrm{~cm}$ above the manometrically determined proximal border

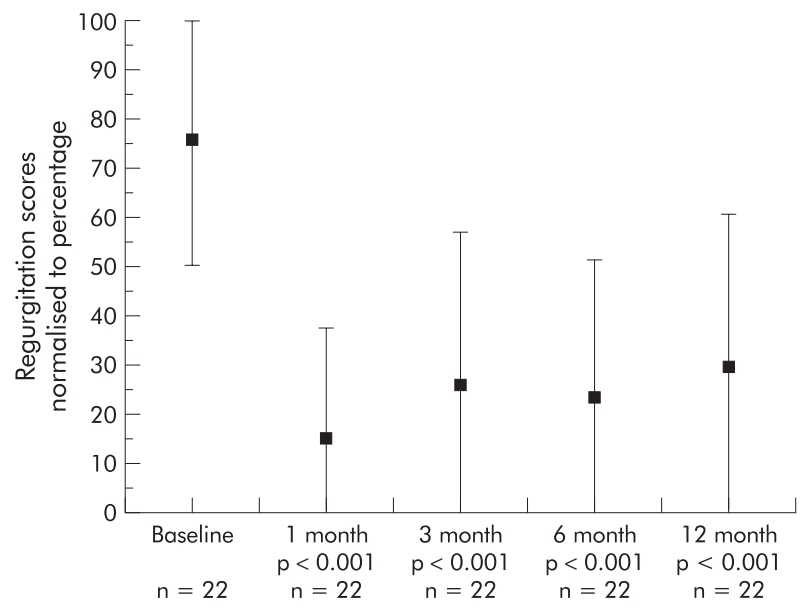

Figure 2 Regurgitation score before (baseline) and one, three, six, and 12 months post procedure. Data are normalised to percentage where a maximum regurgitation score of 3 (that is, severe, daily) is represented as $100 \%$. The square symbol represents the mean and the error bars the SD of the mean. 
Table 2 Pre and three month post procedure means for 24 hour oesophageal $\mathrm{pH}$ values, lower oesophageal sphincter (LOS) pressure, and LOS lengths

\begin{tabular}{|c|c|c|c|c|c|}
\hline \multirow{2}{*}{$\begin{array}{l}\text { Measurement } \\
\text { DeMeester acid score }\end{array}$} & \multirow{2}{*}{$\begin{array}{l}\mathrm{n}^{*} \\
21\end{array}$} & \multicolumn{2}{|l|}{ Baseline } & \multirow{2}{*}{$\begin{array}{l}3 \text { months } \\
33.32(4.73)\end{array}$} & \multirow{2}{*}{$\frac{p \text { Valuet }}{0.028}$} \\
\hline & & 44.1 & (4.338) & & \\
\hline DeMeester acid score for grou & & & & & \\
\hline At 12 months & 13 & 41.36 & (5.59) & $27.28(5.71)$ & 0.039 \\
\hline \multicolumn{6}{|c|}{ DeMeester acid score for group on PPls } \\
\hline At 12 months & 8 & 48.04 & (7.05) & $42.06(7.54)$ & 0.374 \\
\hline Upright (\%) & 21 & 13.47 & $(1.26)$ & $9.09(1.14)$ & 0.002 \\
\hline No of episodes of acid reflux & 21 & 177.35 & (14.03) & $118.2 \quad(13.42)$ & 0.002 \\
\hline Supine (\%) & 21 & 8.17 & $(2.02)$ & $7.06(1.7)$ & NS \\
\hline Total time $\mathrm{pH}<4(\%)$ & 21 & 11.11 & (1.03) & $9.34(1.36)$ & NS \\
\hline LOS pressure $(\mathrm{mm} \mathrm{Hg})$ & 21 & 10.78 & (1.05) & $10.84(0.99)$ & NS \\
\hline LOS length $(\mathrm{cm})$ & 21 & 3.913 & $(0.23)$ & $3.45(0.21)$ & NS \\
\hline
\end{tabular}

Values are mean (SEM).

* Number of patients (one patient excluded as failed to attend for follow up $\mathrm{pH}$ post procedure).

†Wilcoxon signed rank test.

of the OGJ. This was connected to a Mark III Digitrapper (Medtronic, Copenhagen) and recorded for a minimum of 20 hours. Diet was unrestricted but patients were asked to refrain from alcohol for the duration of the study. Standard parameters were recorded including total percentage of time $\mathrm{pH}<4$, upright and supine percentages, total number of episodes, and DeMeester acid score.

\section{Endoscopic plication procedure}

The BARD Endocinch-I endoscopic suturing system was used. This includes a suturing capsule that is attached to the endoscope, a knot pusher, tags attached to sutures, a tag pusher, and a suture cutter. The intention was to place two stitches separated horizontally on the lesser curve by $1 \mathrm{~cm}$. A knot, which brought the two stitches together, was formed of six half hitches creating a plication. Procedure time was recorded from the patient entering the endoscopy room to removal of the endoscope after inspection of the plications in situ at the end of the procedure. Two plications were placed along the lesser curve, in each patient $2 \mathrm{~cm}$ and $1 \mathrm{~cm}$ below the " $Z$ " line. The procedure used is as described previously. ${ }^{15}$ Patients were sedated with a combination of midazolam and pethidine, which was administered intravenously as required throughout the procedure. Oxygen saturation was monitored by pulse oximetry and was maintained above $90 \%$ by $2-4$ litres of oxygen given by nasal catheters.

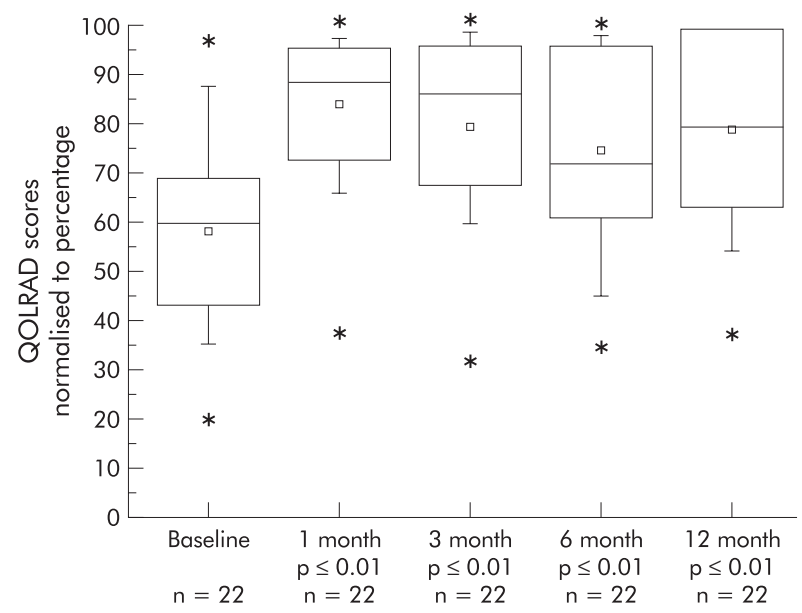

Figure 3 Combined results of the five parameters of quality of life (QOL) assessment before (baseline) and one, three, six, and 12 months post procedure. Data are normalised to percentage where a maximum symptom score of 175 (that is, normality) is represented as $100 \%$. Boxes and symbols as for fig 1 .
Preliminary training in the use of the Endocinch techniques was obtained by attending Professor Paul Swain's laboratory on two occasions and by practising on live pig models at three separate times.

The study design and protocol for obtaining informed consent were passed by the Joint Ethics Committee of St James's Hospital and AMNCH Tallaght Hospital, which is approved by the Minister for Health in Ireland.

Adverse events were defined as any undesirable clinical occurrence experienced by a patient whether device or procedure related.

\section{Statistical analysis}

Statistical analysis was carried out using the StatView package (Abacus Concepts Inc.). The Wilcoxon rank signed test was used to test the significance of $\mathrm{pH}$ and manometry data and symptom scores. A p value $<0.05$ was considered significant.

Graphs were produced by the methods described in the legends to the figures.

\section{RESULTS}

The procedure was performed in 26 patients. Four patients were lost to follow up, two for personal reasons, one left the country, and one because she became pregnant. Twenty two patients completed their one year follow up. Mean age was 39 years (range 22-62); 13 males and nine females. Mean BMI

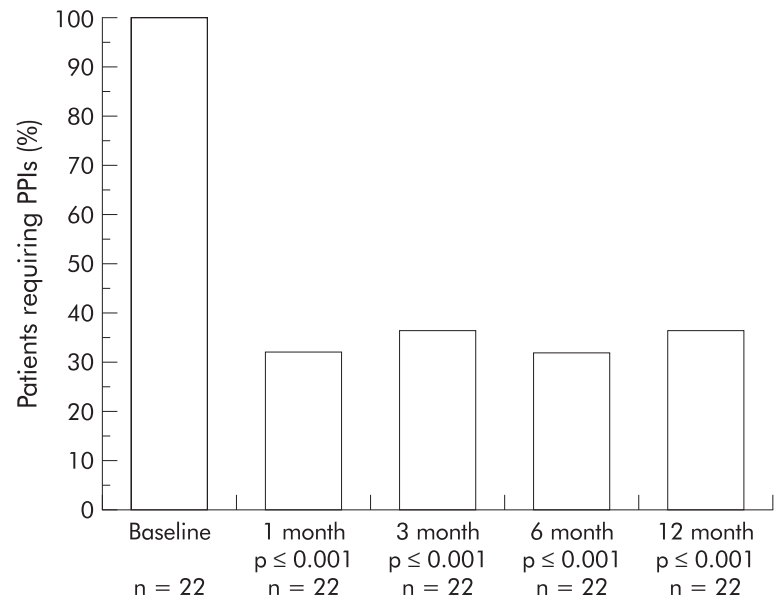

Figure 4 Patient requirements for proton pump inhibitors (PPIs) before (baseline) and one, three, six, and 12 months post procedure (patient off medication was defined as if requiring less than four doses a month). 

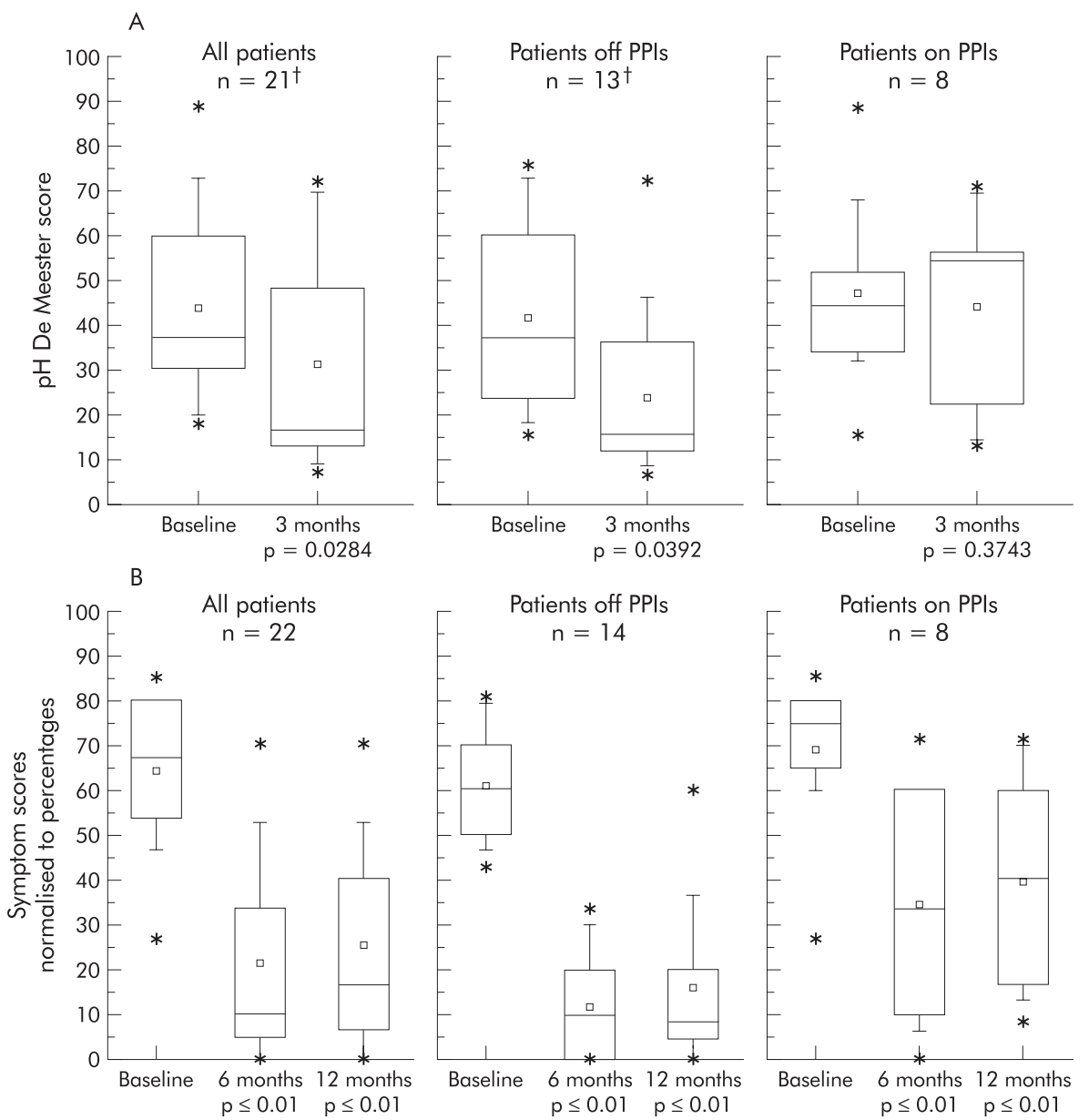

Figure 5 Symptom scores at six and 12 months, and DeMeester scores at three months post procedure in all patients, in patients who reverted back to proton pump inhibitor (PPI) therapy, and in those who remained off PPIs. †One patient failed to attend for follow up 24 hour oesophageal $\mathrm{pH}$ but remained off PPIs at 12 months post procedure. Boxes for symbols as for fig 1 .

was $26 \mathrm{~kg} / \mathrm{m}^{2}$ (range 21-34). All patients received conscious sedation consisting of midazolam (mean dose $21 \mathrm{mg}(8-60)$ ) and pethidine (mean dose $108 \mathrm{mg}$ (25-200)). Median procedure time was 45 minutes (range 25-100).

\section{Post procedure assessments}

\section{Endoscopic surveillance}

All subjects underwent repeat upper gastrointestinal endoscopy at three months post procedure. The plications were found to be in the same position as recorded at the time of the procedure in 17 patients, in three patients one of the two plications had vanished, and in two patients both plications had gone. There was no evidence of worsening of oesophagitis in any of the patients at this stage.

\section{Symptom analysis post procedure}

There was significant improvement in the heartburn symptom score (frequency $\times$ severity) (fig 1 ) and regurgitation frequency (fig 2 ) at one, three, six, and 12 months post procedure.

\section{Manometry and 24 hour pH study}

$\mathrm{pH}$ values were compared before and three months post procedure (table 2 ). Twenty one patients were studied. One patient failed to attend for follow up $\mathrm{pH}$ but he completed the rest of the one year follow up protocol. Mean DeMeester acid score showed significant improvement at three month post procedure (table 2 ). The procedure was also especially effective in the reduction of the percentage upright $\mathrm{pH}$ values and the number of reflux episodes occurring in 24 hours. Manometric studies revealed no significant difference in pre and post procedure LOS pressures and lengths.

\section{Quality of life assessment}

There was significant improvement in all five quality of life parameters, which included emotional distress, sleep disturbances, food/drink problems, physical/social functioning, and vitality, at one, three, six, and 12 months post procedure compared with baseline (fig 3).

\section{Proton pump inhibitor consumption}

Pre procedure, all patients were by definition taking PPIs. The requirement for PPI reduced from $100 \%$ to $36 \%$ after six and 12 months of follow up (fig 4). To assess the relevance of restarting PPIs, the symptoms of those who did/did not revert to PPI intake was determined (fig 5). Those who restarted PPI therapy had significantly more symptoms than those who did not, and a higher oesophageal acid DeMeester score.

\section{Complications of the procedure}

Immediately post procedure, transient complaints occurred (table 3); these included sore throat, vomiting, abdominal pain, chest soreness, bloating, and mild dysphagia. All of these complaints resolved spontaneously within 72 hours. Two patients had significant bleeds; one received a unit of blood. Both were retained overnight for observation. One patient had a gastric mucosal tear which required no further attention. Patients were otherwise routinely discharged from the department after four hours of observation. They were 
Table 3 Summary of complaints

\begin{tabular}{ll}
\hline & No of patients \\
\hline Minor adverse effects & 7 \\
Sore throat & 2 \\
Vomiting & 3 \\
Abdominal pain & 5 \\
Chest soreness & 2 \\
Dysphagia & 2 \\
Bloating & 1 \\
Significant events & 2 Gastric mucosal tear \\
Bleeding & \\
\hline *Required overnight observation. & \\
&
\end{tabular}

instructed to take fluids only for the following 12 hours; thereafter they resumed a normal diet.

\section{DISCUSSION}

This prospective study of the BARD Endocinch-I endoscopic suturing system has demonstrated that it is an effective and safe technique for the management of patients with GORD symptoms who are either dependent on or are not satisfactorily controlled by long term therapy with PPI drugs. The procedure can be performed on an outpatient basis using conscious sedation. These findings are similar to those reported by Swain and colleagues ${ }^{18}$ and Filipi and associates. ${ }^{15}$ In the last 18 months the technique has been used in multiple centres in both the USA and Europe (as well as Canada, South and Central America, Australia, and India).

Following a detailed follow up over a one year period after the procedure, patients noted a significant reduction in the frequency and severity of their heartburn and volume regurgitation symptoms. They had a markedly improved quality of life and a significant reduction in the necessity to take PPIs.

This is the first study to report on the effect of the procedures over a one year follow up. In particular, it shows that improvement in the quality of life of the patient is maintained throughout this period, with no evidence of decline at the end of the year (fig 3). Similarly, requirement for PPIs showed no evidence of increasing over this period (fig 4). These improvements, which compare with their QOLRAD assessment pre procedure while on PPIs, were maintained over a one year period while either not taking PPIs or taking a significantly lower dose of PPIs. Whether or not these improvements will be maintained, only time will tell, especially in the light of the long term follow up findings of Spechler and colleagues ${ }^{13}$ who showed that both medical and surgically treated patients tended to equate over a five year period. However, evidence of sustained improvement at 12 months is encouraging for the procedure's durability, especially in view of the long term follow up findings of Spechler and colleagues. ${ }^{13}$

This study was performed using the BARD Endocinch-I device which required the placing of six half hitches to secure a plication. Since July 2001 the BARD Endocinch-2 device has been available which uses placement of a clip device to secure the plications. In our hands this has significantly reduced the time of the procedures. We have simply used two plications placed vertically along the lesser curve in this study. The technique however is open to variation in the number and site of placement of the plications which have the potential to considerably improve the clinical and technical results.

The three month follow up oesophageal pH study demonstrated that the degree of overall acid reflux over 24 hours was significantly reduced (table 2 ). This was particularly true when patients were in the upright position; the latter being the position that is most commonly associated with significant reflux in our patients (table 2 ). There was also a significant reduction in the overall number of reflux events. These findings are similar to those reported elsewhere. ${ }^{15}$ However, unlike Swain and colleagues, ${ }^{18}$ we did not find evidence that the procedure significantly affected the length or pressure of the LOS. Thus whatever effect the procedure is having on LOS function to reduce the degree of oesophageal acid reflux in these patients, it is too subtle to be identified by the techniques we are presently using to monitor LOS activity.

It may be that the benefit of the procedure is derived simply by decreasing the number and duration of transient lower oesophageal sphincter relaxation (TLOSR) events, an excessive number and size of which characterise the patient with reflux disease ${ }^{19}$ - that is, bringing the number and size of TLOSRs back within the normal range. This may be achieved simply by improving the OGJ flap valve, as suggested by Hill and colleagues. ${ }^{20}$

In a non-randomised prospective study, we compared the results of the BARD Endocinch technique reported here with those obtained using the LNF method. Comparable improvement in symptom score, reduction in PPI intake, and QOL assessments were obtained with both techniques. In the LNF group, the physiological control of oesophageal acid reflux was significantly better than in the Endocinch patients but this occurred at the expense of a higher incidence of post procedure complications. ${ }^{21}$

The marked improvement in patient GORD symptoms, QOLRAD, and reduced requirements for PPIs using the BARD Endocinch technique was associated with some continuing oesophageal acid reflux although in 10 patients the post procedure acid DeMeester score returned to normal levels. As demonstrated in fig 5, those patients who have not had to revert to PPIs at 12 months had a significantly lower degree of acid reflux at three months than those who restarted PPI therapy. This suggests that the degree of control of acid reflux obtained with this technique will be an important aspect of the success or otherwise of the procedure, which does not support findings reported elsewhere. ${ }^{22}$

The subsequent use of PPIs following the procedure was found to be an excellent criterion of the overall success of the procedure, as demonstrated in fig 5 . Even those who restarted on PPIs had significant improvement in their symptoms post procedure but this in turn was significantly less than the value obtained by those who did not revert to taking PPIs. Those who did not restart on PPIs demonstrate just how effective this technique can be. The reason why some patients reverted to PPIs therapy at this stage is unclear but it many relate to the higher acid DeMeester score in this group pre procedure. It is possible that a third plication might improve the clinical and technical results in patients with higher baseline DeMeester scores.

On occasion, patients experience temporary discomfort over the first 48 hours which may take the form of abdominal or chest pain, sore throat, transient mild dysphagia, vomiting, or bloating, but such symptoms have always resolved within 72 hours. There are no reports of serious side effects such as perforation. Haemorrhage can occur during the procedure but usually resolves when the two stitches are brought together as a plication. On one occasion we considered that the duration of bleeding was such as to require the transfusion of a single unit of blood, and in one patient haemorrhage stopped with submucosal injection of adrenaline. On both occasions we observed the patient over night and they were discharged well the following day. On another occasion the needle and tag pusher failed to retract after insertion of the stitch, which caused a gastric mucosal tear on withdrawal of the scope. However, this did not cause any significant morbidity. Vomiting was a problem in two patients during the early days of our experience with the procedure. This may have been related to the prolonged duration of the procedure which 
resulted in a relatively large amount of conscious sedation at that period. However, both modification of this technique by using a clip rather than knots to form a plication, and improved operator technique, has significantly reduced the amount of conscious sedation required.

In conclusion, the BARD Endocinch endoscopic suturing device is an effective and safe alternative method of managing GORD when contrasted with other antireflux therapies. It is associated with minimal discomfort. It significantly reduced 24 hour oesophageal $\mathrm{pH}$ at three months post procedure but only in $48 \%$ did the acid score return to within the normal range. At one year follow up, it continued to significantly reduce heartburn frequency and severity, regurgitation, and to reduce the requirements for antisecretary medications. It also produced significant improvement in all parameters of quality of life measured over the year. This procedure may fill a gap between medical and surgical treatment of GORD. At this stage prospective randomised studies are needed to compare it with both medical and surgical antireflux therapies. Further investigations are also required to assess the number of and where best to place the plications to obtain the best patient care outcome. Continued follow up of patients to assess the long term durability of patient relief from GORD symptoms without the need for PPI therapy is necessary.

\section{ACKNOWLEDGEMENTS}

We are grateful to CR Bard for part funding of this study, to Ms Valerie Trimble for management of patient follow up, and to the staff and the Directorate of the Endoscopy unit at St James's Hospital.

\section{Authors' affiliations}

Z Mahmood, Q Arfin, D G Weir, Department of Clinical Medicine, Trinity College, St James's Hospital, Dublin, Ireland

B P McMahon, Department of Medical Physics and Clinical Engineering, $\mathrm{AMNCH}$, Dublin

P J Byrne, J V Reynolds, Department of Surgery, Trinity College, St James's Hospital, Dublin, Ireland

E M Murphy, Department of Medical Physics and Bioengineering, Trinity College, St James's Hospital, Dublin, Ireland

Conflict of interest: The authors have been the principal teachers of the Endocinch technique for BARD in Europe over the past two years.

\section{REFERENCES}

1 Stanciu C, Bennett JR. Oesophageal acid clearing: one factor in the production of reflux oesophagitis. Gut 1974;15:852-7.

2 Spechler SJ. Epidemiology and natural history of gastro-oesophageal reflux disease. Digestion 1992;51(suppl 1):24-9.
3 Lind T, Havelund T, Carlsson R, et al. Heartburn without oesophagitis: efficacy of omeprazole therapy and features determining therapeutic response. Scand J Gastroenterol 1997;32:974-9.

4 Winters C Jr, Spurling TJ, Chobanian SJ, et al. Barrett's esophagus. A prevalent, occult complication of gastroesophageal reflux disease. Gastroenterology 1987;92:118-24.

5 DeVault KR, Castell DÓ. Guidelines for the diagnosis and treatment of gastroesophageal reflux disease. Practice Parameters Committee of the American College of Gastroenterology. Arch Intern Med 1995; 155:2165-73.

6 Cameron AJ, Ott BJ, Payne WS. The incidence of adenocarcinoma in columnar-lined (Barrett's) esophagus. N Engl J Med 1985;313:857-9.

7 Dimenas E, Glise H, Hallerback B, et al. Quality of life in patients with upper gastrointestinal symptoms. An improved evaluation of treatment regimens? Scand J Gastroenterol 1993;28:681-7.

8 Moayyedi P. Counting the cost of proton pump inhibitors. Gut 2001;49:462-3

$9 \mathbf{O}^{\prime}$ Connor JB, Provenzale D, Brazer S. Economic considerations in the treatment of gastroesophageal reflux disease: a review. Am J Gastroenterol 2000;95:3356-64.

10 Klinkenberg-Knol EC, Meuwissen SG. Treatment of reflux oesophagitis resistant to H2-receptor antagonists. Digestion 1989;44(suppl 1):47-53.

11 Gotley DC, Smithers BM, Rhodes M, et al. Laparoscopic Nissen fundoplication-200 consecutive cases. Gut 1996;38:487-91.

12 Bittner HB, Meyers WC, Brazer SR, et al. Laparoscopic Nissen fundoplication: operative results and short-term follow-up. Am J Surg 1994; 167:193-8.

13 Spechler SJ, Lee E, Ahnen D, et al. Long-term outcome of medical and surgical therapies for gastroesophageal reflux disease: follow-up of a randomized controlled trial. JAMA 2001;285:2331-8.

14 Swain CP, Kadirkamanathann SS, Brown G, et al. Sewing at flexible endoscopy in human gastrointestinal tract. Gastrointest Endosc 1994;40:AB35.

15 Filipi CJ, Lehman GA, Rothstein RI, et al. Transoral, flexible endoscopic suturing for treatment of GERD: a multicenter trial. Gastrointest Endosc 2001;53:416-22

16 Wiklund IK, Junghard O, Grace E, et al. Quality of life in reflux and dyspepsia patients. Psychometric documentation of a new disease-specific questionnaire (QOLRAD). Eur J Surg Suppl 1998:583:41-9.

17 DeMeester TR, Wang Cl, Wernly JA, et al. Technique, indications, and clinical use of 24 hour esophageal $\mathrm{pH}$ monitoring. J Thorac Cardiovasc Surg 1980;79:656-70.

18 Swain CP, Kadairkamanathan SS, Gong F, et al. Endoscopic gastroplasty for gastro-esophageal reflux disease. Gastrointest Endosc 1997;45:AB242.

19 Holloway RH. The anti-reflux barrier and mechanisms of gastro-oesophageal reflux. Baillieres Best Pract Res Clin Gastroenterol 2000; 14:681-99.

20 Hill LD KR, Kraemer SJM, Aye RW, et al. The gastroesophageal flap valve: In vitro and in vivo observations. Gastrointest Endosc 1996;44:541-7.

21 Mahmood Z, Byrne PJ, McCullouch J, et al. A comparison of Bard Endocinch transoesophageal endoscopic plication (BETEP) with laproscopic nissen fundoplication (LNF) for the treatment of gastroesophageal reflux disease (GORD). Gastrointest Endosc 2002;55:463.

22 Pazwash H, Nicholas MG, James GR, et al. Does the DeMeester score predict an improvement in regurgitation following endoluminal gastroplication in gastroesophageal reflux disease ? Am J Gastroenterol 2001;96:AB90. 